\title{
NUMERICAL STUDY OF THE GEOMETRIC INFLUENCE OF A FIN IN A CYLINDRICAL HEAT EXCHANGER FOR MELTING OF PCM
}

\begin{abstract}
F. C. Spengler,
B. Oliveira,

R. C. Oliveski, and L. A. O. Rocha

Universidade do Vale do Rio dos Sinos

Departamento de Engenharia Mecânica

Centro Politécnico

Av. Unisinos, 950 - Cristo Rei, São Leopoldo

Rio Grande do Sul, Brasil fernandocspengler@gmail.com brunooliveira@edu.unisinos.br decesaroo@gmail.com laorocha@gmail.com

Received: February 28, 2019

Revised: March 20, 2019

Accepted: April 11, 2019

\section{ABSTRACT}

The thermal heat storage it's an effective way to suit the energy availability with the demand schedule. It can be stored in the means of sensible or latent heat, the latter applying a material denominated Phase Change Material (PCM), which is provided as organic compounds, hydrated salts, paraffins, among others. The latent heat storage systems offer several advantages, like the practically isothermal process of loading and unloading and the high energy density. However, the low thermal conductivity makes the cycle prolonged on these systems, restricting its applicability. Applying computational fluid dynamics, the behavior of the PCM melting process was studied in cylindrical cavities with horizontal and vertical fins, aiming the optimization of the fin geometry. In this way the fin area was kept constant, varying its aspect ratio. The numerical model was validated with results from the literature and it's composed of the continuity, momentum and energy equations increased by the phase change model. Qualitative and quantitative results are presented, referring to mesh independence, contours of velocity, net fraction and temperature at different moments of the process. The results of the study indicate that the position of the fin in the heat exchanger influences the melting process, although the vertical fins have a faster total melting process, horizontal fins can reach larger partial liquid fractions in less time in the heat exchanger. Such as the position of the fin, the increase of its length propitiates the reduction of the melting time, evidencing the optimal aspect ratio.

Keywords: PCM; CFD; geometric influence
\end{abstract}

\section{NOMENCLATURE}

A area, $\mathrm{m}^{2}$

a fin width, $m$

b fin length, $m$

C mushy zone constant, kg. $\mathrm{m}^{-3} \cdot \mathrm{s}^{-1}$

Cp specific heat, $\mathrm{J} \mathrm{kg}^{-1} \cdot \mathrm{K}^{-1}$

$\mathrm{D}$ diameter, $\mathrm{m}$

DR ray difference, $m$

dt time step, $\mathrm{s}$

e error

Fo Fourier number

g gravity, m.s ${ }^{-2}$

GCI grid convergence index

h heat transfer coefficient, W. $\mathrm{m}^{-2} \cdot \mathrm{K}^{-1}$

$\mathrm{hr} \quad$ representative mesh size

$\mathrm{k}$ thermal conductivity, $\mathrm{W} \cdot \mathrm{m}^{-1} \cdot \mathrm{K}^{-1}$

L latent heat, J.kg-1

Lc characteristic length, $m$

m mass, $\mathrm{kg}$

$\mathrm{N}$ number of computational elements

$\mathrm{Nu} \quad$ Nusselt number

$\mathrm{p}$ pressure, $\mathrm{Pa}$

pg GCI variable

Q heat transfer rate, $\mathrm{W}$

q" heat flux, W.m ${ }^{-2}$

qg GCI variable
$\mathrm{R}$ radius, $\mathrm{m}$

RA aspect ratio

$\mathrm{Ra}$ Rayleigh number

$\mathrm{r}$ refinement level

S source term, Pa.m ${ }^{-1} ; \mathrm{W} . \mathrm{m}^{-3}$

sg GCI variable

Ste Stefan number

$\mathrm{T}$ temperature, $\mathrm{K}$

$\mathrm{t}$ time, $\mathrm{s}$

$\mathrm{V}$ velocity, $\mathrm{m} . \mathrm{s}^{-1}$

$\vec{V} \quad$ velocity vector, $m . s^{-1}$

$\mathrm{x} \quad$ position in the $\mathrm{x}$ direction, $\mathrm{m}$

$\mathrm{y}$ position in the $\mathrm{y}$ direction, $\mathrm{m}$

\section{Greek symbols}

$\begin{array}{ll}\alpha & \text { thermal diffusivity, } \mathrm{m}^{2} . \mathrm{s}^{-1} \\ \beta & \text { liquid fraction } \\ \gamma & \text { volumetric fraction } \\ \Delta & \text { variation } \\ \varepsilon & \text { numeric constant } \\ \zeta & \text { thermal expantion coefficient, } \mathrm{K}^{-1} \\ \lambda & \text { especifc enthapy, } \mathrm{J} . \mathrm{kg}^{-1} \\ \mu & \text { diynamic viscosity, } \mathrm{kg} \cdot \mathrm{m}^{-1} \cdot \mathrm{s}^{-1} \\ \rho & \text { density, kg.m } \\ \varphi & \text { GCI representative value } \\ \phi & \text { areas ratio }\end{array}$


v kinematic viscosity, $\mathrm{m}^{2} \cdot \mathrm{s}^{-1}$

\section{Subscripts}

$\begin{array}{ll}\text { a } & \text { approximate } \\ \text { c } & \text { cross section } \\ \text { ex } & \text { external } \\ \text { ext } & \text { extrapolated } \\ \text { f } & \text { fin } \\ \text { i } & \text { element } \\ \text { in } & \text { internal } \\ \text { l } & \text { liquid } \\ \text { L } & \text { latent } \\ \text { m } & \text { melting } \\ \text { min } & \text { minimum } \\ \text { ref } & \text { reference } \\ \text { s } & \text { solid } \\ \text { se } & \text { sensible } \\ \text { w } & \text { wall }\end{array}$

\section{INTRODUCTION}

With the energy crisis of the 1970s, studies have come about ways of storing energy. Therefore, phase change materials (PCM) have come to light, resulting in research on its behavior and application forms (Agyenim et al., 2010). The studies remain in vogue, being approached in an experimental and numerical way.

The influence of thermal and geometric parameters of PCM systems has been the focus of studies, such as the addition of heat transfer using fins, as presented by Agyenim, Eames and Smyth (2009), longitudinal fins have superior efficiency than circular ones, both in loading and unloading processes. While Wang et al. (2016) demonstrated the influence of the fin arrangement in a circular cavity, revealing superior efficiency for angles between $60^{\circ}$ and $90^{\circ}$. The effect of different slopes was also addressed by Kamkari and Shokouhmand (2014), with the slope of a rectangular cavity filled with lauric acid PCM being varied, a significant influence was recorded on the melt time and heat transfer rate.

Numerical CFD simulations provide concise results in relation to thermal energy storage systems, as presented by Almsater et al. (2017) which reported that the duration of the melting process is generally faster compared to the solidification process because of natural convection.

Storage systems with fins, which can be interchangeable between vertical and horizontal, enable applications of industrial interest. The objective of this work is to analyze numerically the geometric and positioning influence of internal fins to circular cavities, when submitted to the PCM melting process.

\section{PROBLEM PRESENTATION}

The problem to be studied consists of a heat exchanger composed of an annular section tube, the inner and outer radius of which are respectively $R_{\text {in }}=20 \mathrm{~mm}$ and $R_{e x}=40 \mathrm{~mm}$. The fins have area $\left(A_{f}\right)$, obtained by the product of its length (b) by its thickness (a). The exchanger is filled by lauric acid PCM, initially in the solid state. For this geometry two fins were proposed: horizontally and vertically aligned, according to Fig. 1.
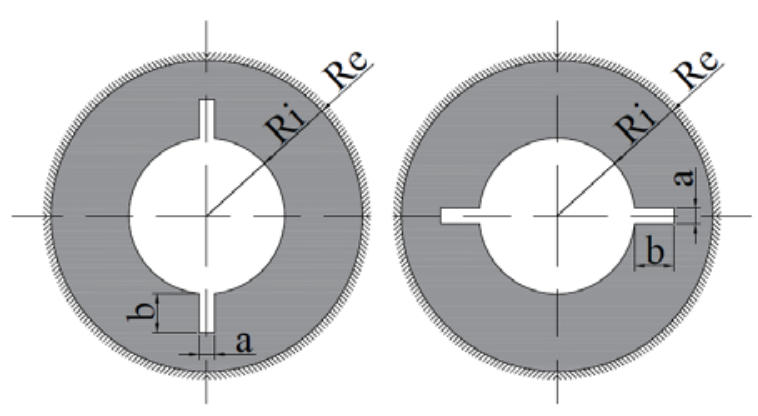

Figure 1. Problem geometries.

The area of the fins $\left(\mathrm{A}_{\mathrm{f}}\right)$ was kept constant and equal to $10 \mathrm{~mm}^{2}$. It was decided to study 5 vertical and 5 horizontal fins. The length (b = DRxRA) of these was obtained considering the following aspect ratios (RA), also predetermined: 1/8, 1/4, 1/2, 3/4 and $7 / 8$, where $D R=R_{e x}-R_{i n}$. Thus, the thickness of the fin (a) was obtained as: $a=A_{f} / b$. The analyzed dimensions are listed in Table 1.

Table 1. Fins dimensions.

\begin{tabular}{|c|c|c|c|c|c|c|}
\hline \multirow{2}{*}{$\phi=0.01$} & $\mathrm{~b}[\mathrm{~mm}]$ & 2.5 & 5 & 10 & 15 & 17.5 \\
\hline & $\mathrm{a}[\mathrm{mm}]$ & 4.00 & 2.00 & 1.00 & 0.67 & 0.57 \\
\hline
\end{tabular}

\section{MATHEMATICAL MODEL}

The mathematical model is presented in the sequence.

$$
\begin{gathered}
\frac{\partial \rho}{\partial t}+\nabla(\rho \vec{V})=0 \\
\frac{\partial \rho \vec{V}}{\partial t}+\nabla(\rho \vec{V} \vec{V})=-\nabla p+\nabla(\mu \nabla \vec{V})+\rho \vec{g}+S \\
S=\frac{(1-\gamma)^{2}}{\left(\gamma^{3}+\varepsilon\right)} C \vec{V}
\end{gathered}
$$

composed of mass (1) and momentum (2) equations, plus the phase change model and the source term(3), where $\rho$ is the density, $\mathrm{t}$ is the time, $\vec{V}$ the vector velocity, $p$ is the pressure, $\mu$ is the dynamic viscosity, $\vec{g}$ the gravity acceleration, $\gamma$ is the liquid fraction during the phase change, $\varepsilon$ is a constant value of 0.001 to avoid division by zero and $\mathrm{C}$ is the constant 
of the porous zone, which depends on the morphology of the porous zone (Voller and Prakash, 1987).

$$
\begin{gathered}
\gamma=\left\{\begin{array}{ccc}
0 & \text { if } & T<T_{s} \\
1 & \text { if } & T>T_{l} \\
\frac{T-T_{s}}{T_{l}-T_{s}} & \text { if } & T_{s}<T<T_{l}
\end{array}\right. \\
\frac{\partial(\rho \lambda)}{\partial t}+\nabla(\rho \vec{V} \lambda)=\nabla(k \nabla T) \\
\lambda_{s e}=\lambda_{\text {ref }}+\int_{T_{\text {ref }}}^{T} C_{p} d T \\
\lambda_{L}=\gamma L
\end{gathered}
$$

where $T_{s}$ and $T_{1}$ are the temperatures of the PCM in the solid and liquid state respectively. The energy conservation is contemplated by the equations (5-7) (Shmueli, Ziskind and Letan, 2010), where $\mathrm{k}$ is the thermal conductivity, $\lambda$ is the total enthalpy, obtained by the sum of the sensitive enthalpy $\left(\lambda_{\mathrm{se}}\right)$ and the enthalpy change in phase change $\left(\lambda_{L}\right)$, where $\lambda_{\text {ref }}$ is the enthalpy at the reference temperature $\left(\mathrm{T}_{\text {ref }}\right), \mathrm{Cp}$ is the specific heat and $\lambda_{\mathrm{L}}$ is a function of the temperature of the liquid fraction in the porous zone, where $\mathrm{L}$ is the latent heat.

\section{NUMERICAL APPROACH}

The computational domain was considered bidimensional and has three control surfaces, which are: fin, internal cylindrical surface and external cylindrical surface, besides the plane of symmetry. While the outer surface is adiabatic, the fin and inner surface have a constant temperature of $80^{\circ} \mathrm{C}$ and the initial temperature of the PCM is $20^{\circ} \mathrm{C}$. All surfaces were considered impermeable and non slip.

The study was performed by Computational Fluid Dynamics (CFD). The software Workbench 18.2 and the modules Design Modeler, Meshing, Fluent and CFD-Post, of ANSYS, was used.

Regarding the control conditions of the simulation, for the pressure-velocity coupling the SIMPLE method was used, the gradient and pressure were defined as Last Squares Cell Based and Presto respectively, while the momentum and energy were established as Second Order Upwind. The relaxation factors used for the pressure, specific mass, field forces, amount of movement, liquid fraction and energy were respectively: $0.3 ; 1 ; 0.5 ; 0.5 ; 0.6$ and 1 . The timestep was $0.01 \mathrm{~s}$, with a limitation of 1000 interactions per interval. In relation to the convergence criteria, $10^{-6}$ was adopted for continuity and velocity, while $10^{-8}$ was adopted for the energy.

The physical properties of the lauric acid were obtained from Kamkari and Shokouhmand, (2014) and Valeri and Meirelles, (1997).

\section{MESH INDEPENDENCE}

Three computational meshes (M1, M2, M3), as presentend in Fig. 2, were created with 28457, 14520 and 10118 respectively. The analysis of mesh independence was performed by the GCI method (Celik et al., 2008), which obtained a maximum value of $1.2241 \%$, while the average value was $0.0026 \%$.

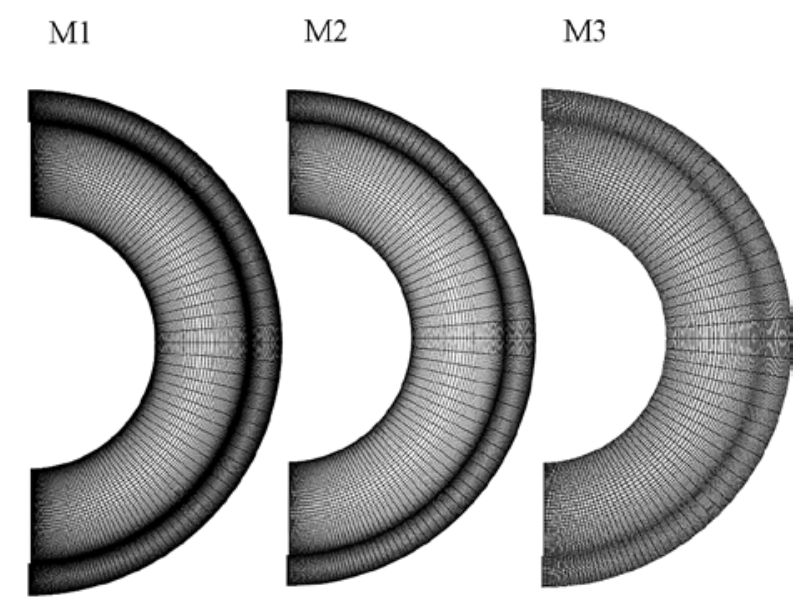

Figure 2. Different refinements of the computational meshes.

\section{NUMERICAL VALIDATION}

For numerical validation were used the results of Yuan et al. (2016), which also consists of an internally finned annular heat exchanger filled with lauric acid PCM.

In the Fig. $3(\mathrm{a}, \mathrm{b})$ are presented liquid fraction fields $(\beta)$ for $\mathrm{t}=1,10,20$ and $60 \mathrm{~min}$, obtained by this paper and by Yuan et al. (2016), respectively. In these figures, the blue region represents the solid phase, while the red region represents the liquid phase of the PCM. There is great similarity between results, both in the initial instants, where there is predominance of the heat conduction process, because it presents a greater amount of solid, and in the instants where there is predominance of the natural convection process, where the liquid phase is more present.

The convective process, mentioned previously, can be observed in Fig. 4, which shows the liquid fraction contours in the left section of the exchanger and the velocity vectors in its right. In the detail of the velocity field, two convective currents can be observed: one ascending and another descending. As the internal radius of the exchanger is heated in relation to the PCM, this causes a reduction of the specific mass of the PCM in that region and consequent buoyancy, which causes the upward convective current. By mass conservation, a descending convective current is also present. 
(a)

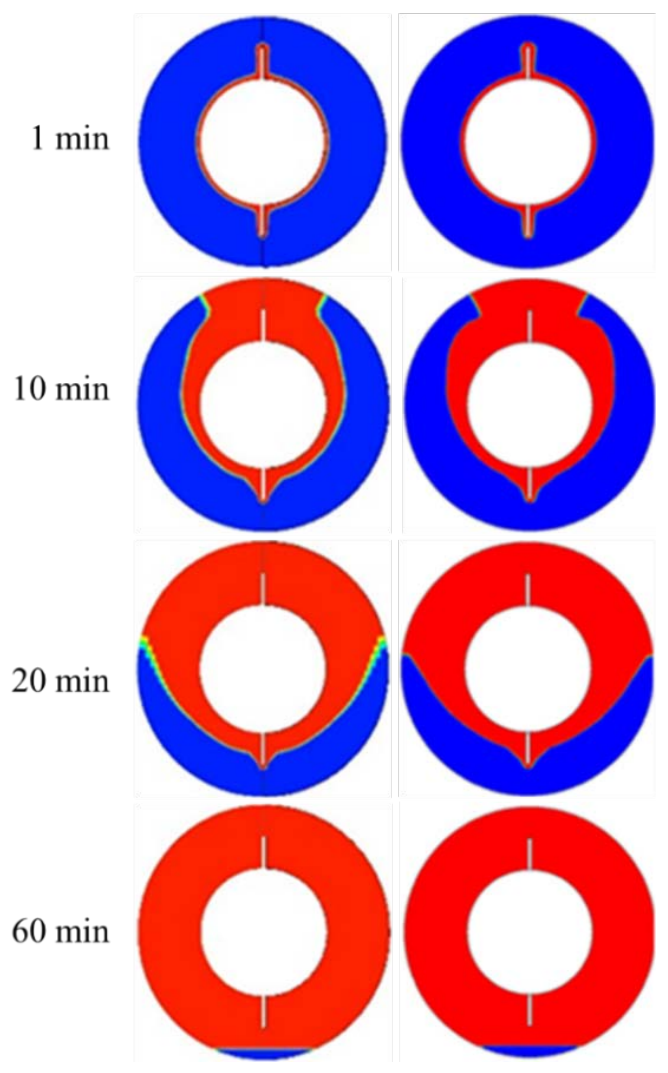

Figure 3. Liquid fraction to $\mathrm{t}=1$, 10, 20 e $60 \mathrm{~min}$ : (a) this paper; (b) Yuan et al., (2016).

Still in relation to the detail of Fig. 4, a welldefined shear region between the two convective currents is also observed. Furthermore, it is observed that the speed profiles are very well defined, both those of the upstream and downstream flow, which indicates good mesh refinement.
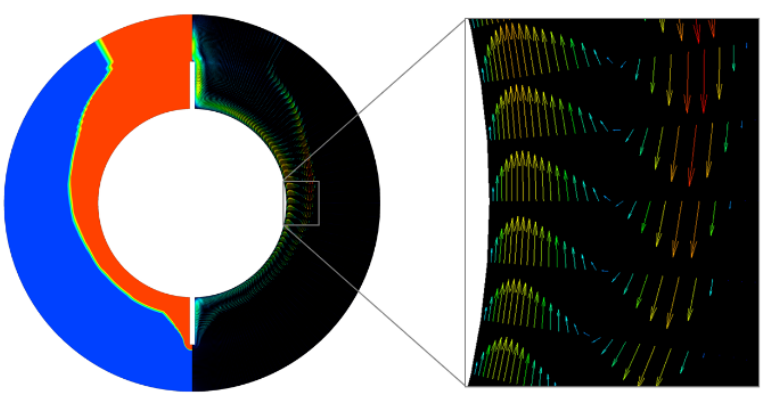

Figure 4. Contours of $\beta$ and vectors of velocity, with detail in the shear region.

In quantitative terms, the numerical validation was performed through the net fraction profile as a function of the Fourier number. The results obtained by the present work and those obtained by Yuan et al. (2016) can be observed in Fig. 5. A good agreement between results is observed. In this case, the average percentage difference was approximately
$2 \%$, and at the end of the process this value reduces to $0.1 \%$. Considering the good results presented in Figs. 3, 4 and 5, the model is considered validated and suitable for Case Study.

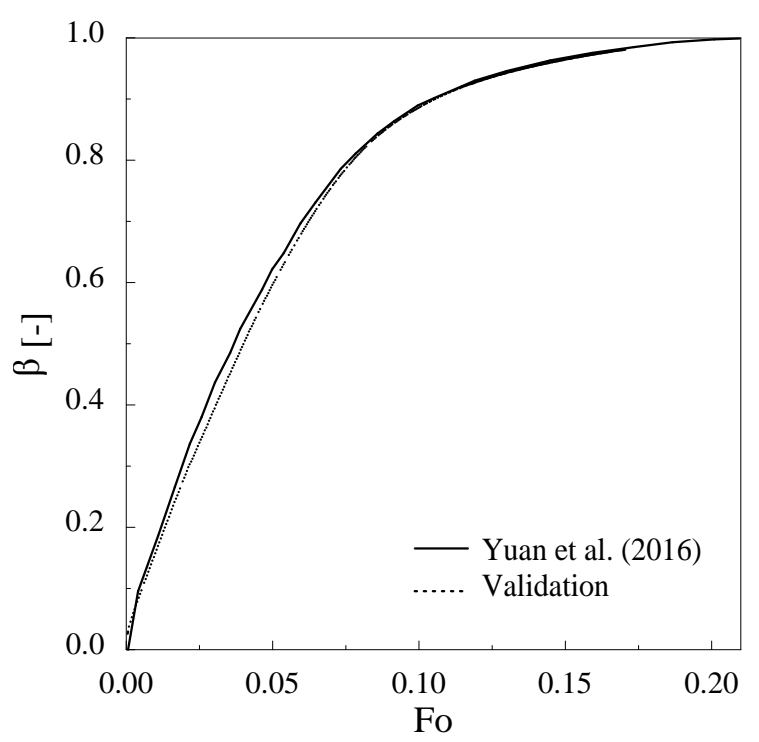

Figure 5. Liquid fraction by Fourrier: Yuan et al. and this paper.

\section{CASE STUDY}

Figure 6 shows liquid fraction profiles as a function of time for 5 vertical fins (RA $=7 / 8,3 / 4$, $1 / 2,1 / 4$ and 1/8) and the same number and dimensions of horizontal fins. Initially it can be observed that, for all cases, up to approximately $100 \mathrm{~s}$, the values of $\beta$ are practically equal. Then the profiles are discriminated, noting the prevalence of melting process for the horizontal fins, being the melting process faster in the fin with aspect ratio 7/8.

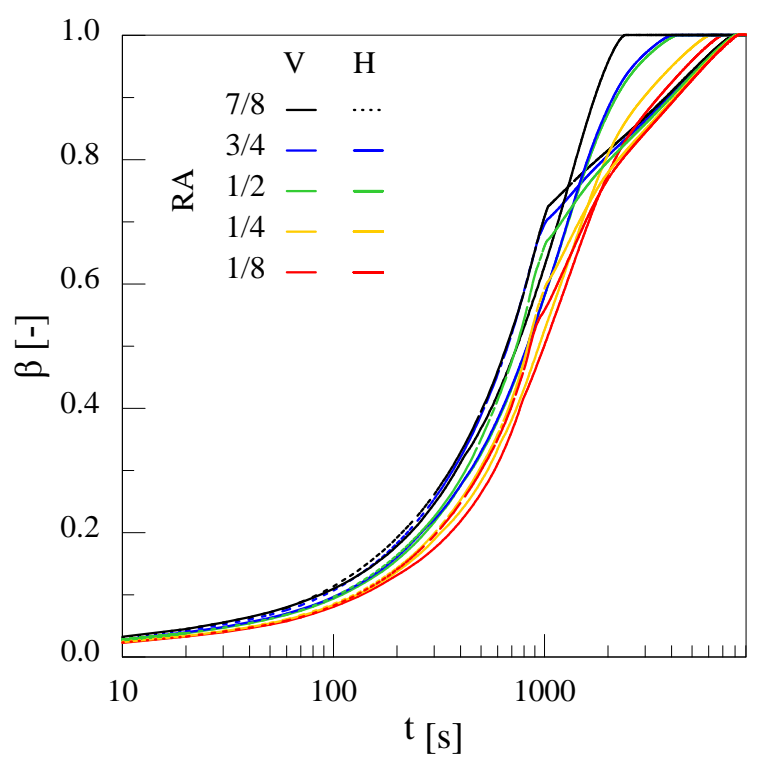

Figure 6. Transient liquid fraction profile for different cases. 
From $\mathrm{t}=1000 \mathrm{~s}$ there is a transition in the behavior of the profiles relative to the horizontal fins, the melting process being slower from this moment. In the seconds that follow, the complete melting of the cases with vertical fins is verified, starting with the case with the highest aspect ratio (RA 7/8) and following in an ordered manner until the fusion of the case with the lowest aspect ratio (RA 1/8). After all cases with vertical fins have reached $\beta=1$, cases with horizontal fins complete the fusion, also in an ordered manner, starting with the case with the highest RA, until the case with the lowest RA.

The processes described up to this point can be contrasted with liquid fraction and temperature contours, which are shown in Fig. 7(a, b, c) for RA of $7 / 8,1 / 2$ and $1 / 8$ respectively for vertical and horizontal fins at the instants 600 and 2400 s. With respect to the instants of the presented fields, the parameter used was the total time of fusion of the fastest case, $2400 \mathrm{~s}$, relative to the case with vertical fin and RA $=7 / 8$ as well as an initial instant (600 s) that allows the visualization of the heat exchange processes involved in the beginning of the melting process. In the center of the cavities is indicated the value of $\beta$.

By contrasting the columns relative to the instant $600 \mathrm{~s}$ with vertical and horizontal fins of Fig. 7, what has already been evidenced in the profiles of Fig. 6 is confirmed, the melted region in cases with horizontal fins is higher than that observed in cases with vertical fins.

Such behavior can be explained by observing the temperature fields at the same instants, whereas cases with vertical fins have relatively stratified regions, indicating only upward flow in the region near the heated wall and fin, the cases concerning the horizontal fins indicate temperature fields diffuse Such behavior is congruent with the convective Rayleigh-Bénard pattern expected on a heated flat surface. Given the greater dynamism of horizontal fin cases, in the initial instants, the fusion process is faster than cases with vertical fins.

Also, in relation to Fig. 7, for $t=2400 \mathrm{~s}$, a change in the behavior in relation to the results in $\mathrm{t}=600 \mathrm{~s}$ is observed, the fusion being faster in cases with vertical fins. Since the solid region is restricted to the lower section of the cylindrical cavity, and the temperature profiles in all cases are stratified, showing little diffuse behavior. However, cases with vertical fins have the particularity of greater proximity to the solid region after this period, unlike the cases with horizontal fins, whose closest heated region is the cylinder wall itself. This fact makes the process of melting the horizontal fins protracted than the cases with vertical fins.

In Fig. 8 it is possible to observe the heat flux profiles over time for all analyzed cases. It is observed that all start with a maximum value of q" higher than $15000 \mathrm{~W} \mathrm{~m}^{-2}$, that gradually decrease until approximately $100 \mathrm{~s}$. (a)
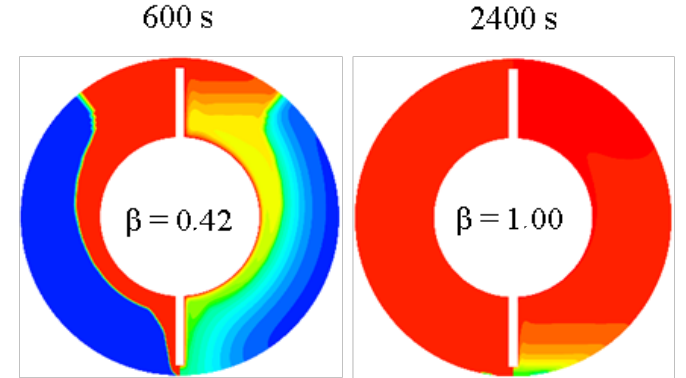

(b)
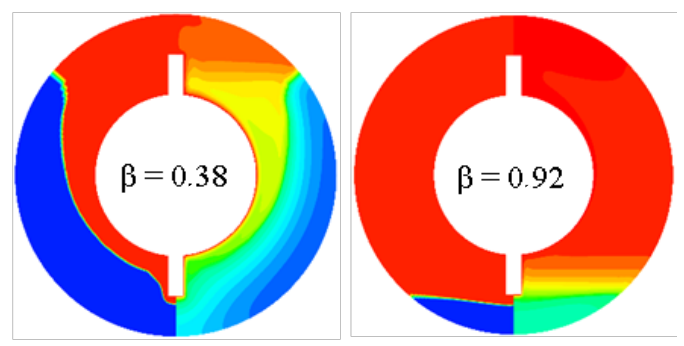

(c)
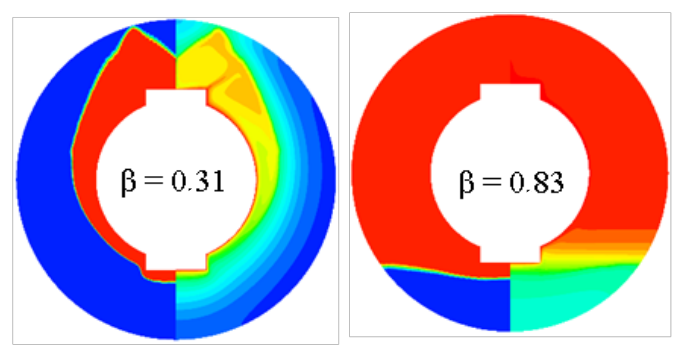

(a)
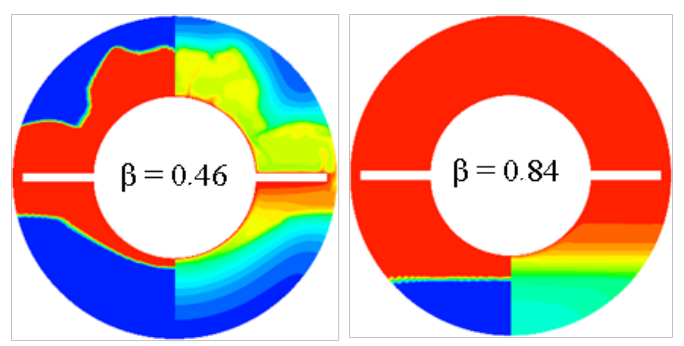

(b)
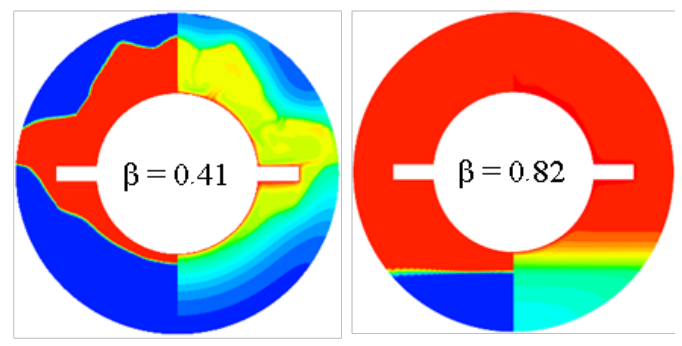

(c)
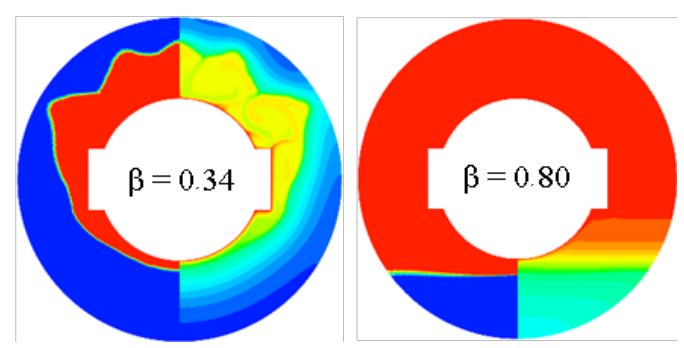

Figure 7. Contours of $\beta$ (left) and T (right), of different moments, for cases with vertical and horizontal fins with RA: (a) 7/8, (b) 1/2 and (c) 1/8.

From this moment the heat flux profiles have small fluctuations up to approximately $1000 \mathrm{~s}$. In this period, the cases that oscillate more are those 
referring to horizontal fins, represented by dashed lines. Such behavior can be correlated with the phenomena previously described, given the greater dynamism of the process, due to the convective Rayleigh-Bénard pattern. From about $1000 \mathrm{~s}$ approximately, there is a marked drop in the value of $\mathrm{q}$ for the cases with horizontal fin, which is due to the end of the diffusive processes active until then, being the transfer of heat restricted to a process practically conductive in molten and stationary liquid. For cases with vertical fins, the value of q" drops gradually, to zero, as the melting approaches the end.

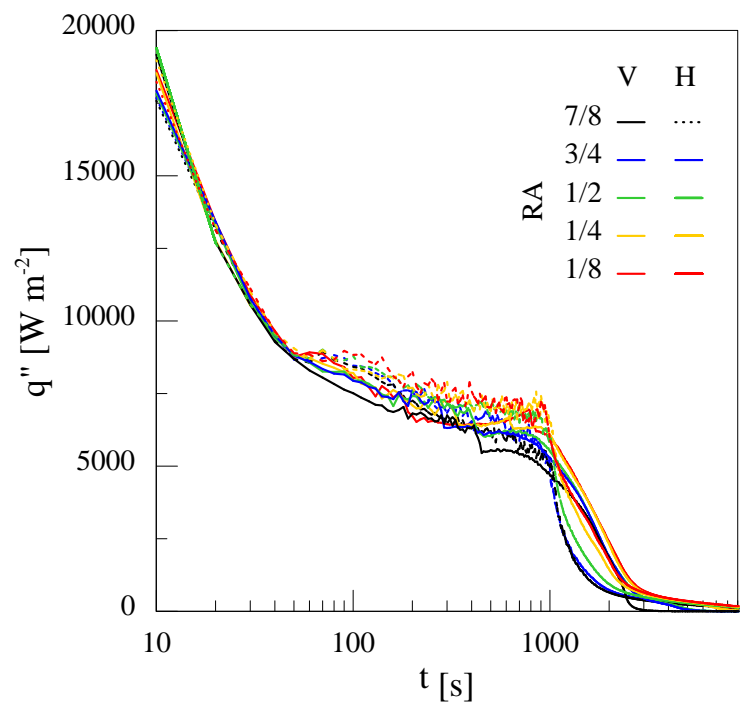

Figure 8. Transient heat flux profile for different cases.

\section{CONCLUSIONS}

The objective of this work was to verify the geometric influence of a fin, in a latent heat exchanger filled with PCM, using numerical computational simulation. For this purpose, commercial software ANSYS Fluent was used. The mathematical model is composed of mass, momentum and energy equations, plus the phase change model and the source term. The computational domain is bidimensional and formed by a refined quadrilateral mesh in the regions with the greatest thermal and fluid dynamics variation.

Validation of the numerical model was performed by comparing velocity fields and mass flow profile with results from the literature, obtaining good results, thus enabling the continuity of the research.

The Case Study results for annular cylindrical section cavities, with vertically or horizontally arranged fins, filled with lauric acid PCM, indicated that:

- although cases with horizontal fins have a more intense melting process at the outset, vertical finned heat exchangers have a faster total melting process;
- the partial and total melting time is lower for fins with higher aspect ratio;

- the geometric and positioning characteristics of the fin significantly influence the melting process in a heat exchanger.

The study carried out offers a study of the optimization of the fin in a heat exchanger, for the lower PCM melting time, for different fin and cavity area relationships, besides the behavior with other PCMs, these suggestions being the continuity of this work.

\section{ACKNOWLEDGEMENTS}

This work was conducted during a scholarship supported by CAPES at the University UNISINOS. Financed by CAPES - Brazil Federal Agency for Support and Evaluation of Graduate Education within the Ministry of Education of Brazil.

\section{REFERENCES}

Agyenim, F., Eames, P., and Smyth, M., 2009, A Comparison of Heat Transfer Enhancement in a Medium Temperature Thermal Energy Storage Heat Exchanger Using Fins, Solar Energy, Vol. 83, No. 9, pp. 1509-1520.

Agyenim, F., Hewitt, N., Eames, P., and Smyth, M., 2010, A Review of Materials, Heat Transfer and Phase Change Problem Formulation for Latent Heat Thermal Energy Storage Systems (LHTESS), Renewable and Sustainable Energy Reviews, Vol. 14, No. 2, pp. 615-628.

Almsater, S., Alemu, A., Saman, W., and Bruno, F., 2017, Development and Experimental Validation of a CFD Model for PCM in a Vertical Triplex Tube Heat Exchanger, Applied Thermal Engineering Journal, Vol. 116, pp. 344-354.

Celik, I. B., Ghia, U., Roache, P. J., Freitas, C. J., Coleman, H., and Raad, P. E., 2008, Procedure for Estimation and Reporting of Uncertainty Due to Discretization in CFD Applications, Journal of Fluids Engineering, Vol. 130, No. 7, pp. 78001-78004.

Kamkari, B., and Shokouhmand, H., 2014, Experimental Investigation of Phase Change Material Melting in Rectangular Enclosures with Horizontal Partial Fins, International Journal of Heat and Mass Transfer, Vol. 78, pp. 839-851.

Valeri, D., and Meirelles, A. J. A., 1997, Viscosities of Fatty Acids, Triglycerides, and their Binary Mixtures, Journal of the American Oil Chemists' Society, Vol. 74, No. 10, pp. 12211226.

Voller, V. R., and Prakash, C., 1987, A Fixed Grid Numerical Modelling Methodology for Convection-Diffusion Mushy Region Phase-Change Problems, International Journal of Heat and Mass Transfer, Vol. 30, No. 8, pp. 1709-1719.

Wang, P., Yao, H., Lan, Z., Peng, Z., Huang, Y., and Ding, Y., 2016, Numerical Investigation of 
PCM Melting Process in Sleeve Tube with Internal Fins, Energy Conversion and Management, Vol. 110, pp. 428-435.

Yuan, Y., Cao, X., Xiang, B., and Du, Y., 2016, Effect of Installation Angle of Fins on Melting Characteristics of Annular Unit for Latent Heat Thermal Energy Storage, Solar Energy, Vol. 136, pp. 365-378. 